\title{
Control of Muscle Fibro-Adipogenic Progenitors by Myogenic Lineage is Altered in Aging and Duchenne Muscular Dystrophy
}

\author{
Claudine Moratala,b Nicole Arrighi ${ }^{a} \quad$ Claude A. Dechesne ${ }^{a} \quad$ Christian Dania \\ aUniversité Côte d'Azur, CNRS, Inserm, iBV, Nice, France, bUniversité Côte d'Azur, Inserm, C3M, Nice, \\ France
}

\author{
Key Words \\ Adipocytes $•$ Myofibroblasts $•$ Muscle progenitors $•$ Myopathies
}

\begin{abstract}
Background/Aims: Fibro-adipogenic progenitors (FAPs), a muscle-resident stem cell population, have recently emerged as important actors of muscle regeneration by interacting with myogenic progenitors (MPs) to promote the formation of new muscle fibers. However, FAPs are also considered as main contributors of intramuscular fibrotic and fat depositions, resulting in a poor quality of muscles and a defective regeneration in aging and Duchenne Muscular Dystrophy disease (DMD). Therefore, the understanding of the control of FAP fate is an important aspect of muscle repair and homeostasis, but little is known in humans. We wondered the extent to which human FAP proliferation, adipogenesis and fibrogenesis can be regulated by human myogenic progenitors (MPs) in physiological and pathological contexts. Methods: FAPs and MPs were isolated from skeletal muscles of healthy young or old donors and DMD patients. FAP/MP contact co-cultures and conditioned-media from undifferentiated MPs or differentiated myotubes were assessed on both proliferation and fibro-adipogenic differentiation of FAPs. Results: We showed that soluble molecules released by MPs activate the phosphoinositide 3-kinase (PI3Kinase)/Akt pathway in FAPs, resulting in the stimulation of FAP proliferation. FAP differentiation was regulated by MP-derived myotubes through the secretion of pro-fibrogenic factors and anti-adipogenic factors. Importantly, the regulation of FAP adipogenic and fibrogenic fates by myotubes was found to be mediated by Smad2 phosphorylation and the gene expression of glioma-associated oncogene homolog 1 (GLI1). Surprisingly, the regulations of proliferation and differentiation were disrupted for FAPs and MPs derived from aged individuals and patients with DMD. Conclusion: Our results highlight a novel crosstalk between FAPs and the myogenic lineage in humans that could be crucial in the formation of adipocyte and myofibroblast accumulation in dystrophic and aged skeletal muscle.




\section{Cellular Physiology Cell Physiol Biochem 2019;53:1029-1045 \\ \begin{tabular}{ll|l} 
and Biochemistry & DOI: 10.33594/000000196 & Published online: 23 December 2019 \\
Cell Physiol Biochem Press GmbH\&Co. KG
\end{tabular} \\ Moratal et al.: Myoblast Effects on Fibro-Adipogenic Progenitors}

\section{Introduction}

Adult skeletal muscle possesses a powerful regenerative capacity that depends on satellite cells which give rise to myogenic progenitors (MPs). Satellite cells reside beneath the basal lamina, closely juxtaposed to the muscle fibers [1]. After injury, quiescent satellite cells become activated, proliferate, and give rise to myoblasts which fuse to existing damaged fibers for repair or among themselves for new myofiber formation. Muscle regeneration is a complex phenomenon that requires a close collaboration between satellite cells and populations such as bone-marrow or muscle-derived stem cells, mesoangioblasts, pericytes and immune cells [2-6].

In the last past decade, a population of fibro-adipogenic progenitors (FAPs) localized between skeletal muscle fibers has been identified in mice $[7,8]$ and in humans by us and other groups [9-14]. When isolated from human muscle, FAPs have an intrinsic adipogenic potential manifested in culture by exposure to adipogenic conditions [10-14] and can also differentiate into fibrogenic cells in response to transforming growth factor $\beta 1$ (TGF $\beta 1$ ) [13, 15]. FAPs are considered as a determinant source of intramuscular adipocyte deposition and fibrosis $[16,17]$. Fibro-adipogenic depositions within muscles are encountered in a variety of physiopathological conditions resulting in significant muscle weakness and decreased passive range of motion [18]. In particular, such depositions can be found in aged muscles $[19,20]$ and are a hallmark of Duchenne muscular dystrophy (DMD) [21].

The knowledge of FAP roles in tissue regeneration is largely based on data obtained in mice. FAPs expand during regeneration at the same time as MPs and secrete paracrine factors that support myoblast differentiation $[7,22]$. The regulation of FAP proliferation and differentiation just begins to be unveiled. During normal repair, FAP expansion is transient and followed by a rapid reduction in their number [7]. This is controlled by macrophages that tightly regulate the balance of pro-apoptotic and pro-survival signals driven by tumor necrosis factor $\alpha(\mathrm{TNF} \alpha)$ and TGF $\beta 1$, respectively [23]. These signals appear in two sequential separate waves in the case of successful regeneration; however, in chronic conditions, such as in dystrophic muscle, this sequence is disrupted, leading to timely overlap of TNF $\alpha$ and TGF $\beta 1$ contradictory signaling $[23,24]$. Also, FAP adipogenesis is inhibited by satellite cellderived muscle fibers [8].

In humans, a pro-mitotic role of platelet-derived growth factor-AA [15] and IL-15 [25] on FAP proliferation was demonstrated, and an anti-adipogenic action of IL-15 [25], Hedgehog signaling [26], TNF $\alpha$ and TGF $\beta$ family members [13] was shown on FAP differentiation. While these studies demonstrate a regulation of FAPs by molecules secreted in regenerating muscles, a direct link between myogenic lineage and FAPs remains to be established in a physiological context and in DMD and aging environment in which FAPs number is disrupted $[15,27]$. Our hypothesis is that, in a non-pathogenic context, MPs control FAPs to prevent the development of adipose and fibrotic deposits that are observed in skeletal muscles of DMD patients and aged donors. Thus, this study aimed to investigate the molecular mechanisms involved in the in vitro regulation of FAP proliferation and differentiation by MP lineage isolated from skeletal muscles of healthy young or aged donors and DMD patients.

\section{Materials and Methods}

\section{Reagents and antibodies}

Cell culture media, serum, buffer, and trypsin were purchased from Lonza (Verviers, Belgique) and cell culture reagents from Sigma-Aldrich Chimie (Saint-Quentin Fallavier, France).

Fluorochrome-conjugated antibodies for flow cytometric analysis were purchased from BD Biosciences (San Diego, USA) (CD56-APC 345812; CD140a-PE 556002).

Monoclonal antibodies for immunofluorescence analysis were purchased as indicated: anti- $\alpha$ SMA (A 5228) from Sigma-Aldrich Chimie, Alexa Fluor 594 goat anti-mouse IgG from Molecular Probes (Eugene, USA). 


\section{Cellular Physiology Cell Physiol Biochem 2019;53:1029-1045 \\ \begin{tabular}{ll|l} 
and Biochemistry & $\begin{array}{l}\text { DOI: 10.33594/000000196 } \\
\text { Published online: 23 December } 2019\end{array}$ & $\begin{array}{l}\text { c } 2019 \text { The Author(s). Published by } \\
\text { Cell Physiol Biochem Press GmbH\&Co. KG }\end{array}$
\end{tabular} \\ Moratal et al.: Myoblast Effects on Fibro-Adipogenic Progenitors}

Antibodies for immunoblot analysis were purchased from Cell Signaling (Ozyme, St Quentin en Yvelines, France): anti-phospho-Akt (Thr308, 9275), anti-Akt (9272), antiphospho-p44/42 (Thr202/ Tyr 204, 9106), anti-p44/42 (9102), anti-phospho-Smad2 (Ser465/467, 3101), antiSmad2/3 (3102). Anti- $\beta$-tubulin antibody was purchased from Sigma-Aldrich.

Human recombinant TGFß1 (100-21) was purchased from Peprotech (Neuilly sur Seine, France) and used at $5 \mathrm{ng} / \mathrm{ml}$. Specific inhibitors wortmannin (W1628) and SB431542 (S4317) were purchased from Sigma-Aldrich Chimie and used at $200 \mathrm{nM}$ and $5 \mu \mathrm{M}$, respectively. Cyclopamine was purchased from Calbiochem (Merck Millipore, Fontenay sous Bois, France) and used at $5 \mu \mathrm{M}$. U0126 was purchased from Promega (Charbonnière-lesBains, France) and used at $10 \mu \mathrm{m}$.
Table 1. Characteristics of healthy young or aged donors and DMD patients. ND: not determined.

\begin{tabular}{|c|c|c|c|c|}
\hline Name & Gender & Age (year) & Muscle origin & Use (figure number) \\
\hline \multicolumn{5}{|c|}{ healthy and young donors } \\
\hline 1 & male & 8 & paravertebral & 1A, 1C, 2F, 3B, 3D, 3E, 3F, 4A, 4B, 4C, 4D, 5D, 6C \\
\hline 2 & male & 1 & paravertebral & 3B, 3D, 3E, 3F, 4A, 4B, 4C, 4D, 5D \\
\hline 3 & male & 4 & nevus left shoulder & $3 \mathrm{~B}, 3 \mathrm{D}$ \\
\hline 4 & male & 1 & gluteus maximus & $1 \mathrm{~A}, 3 \mathrm{~B}, 3 \mathrm{D}$ \\
\hline 5 & male & 17 & gluteus maximus & $1 \mathrm{~A}, 1 \mathrm{~B}, 1 \mathrm{C}, 2 \mathrm{~F}, 3 \mathrm{~B}, 3 \mathrm{D}, 3 \mathrm{E}, 3 \mathrm{~F}, 4 \mathrm{~A}, 4 \mathrm{~B}, 4 \mathrm{C}, 4 \mathrm{D}, 5 \mathrm{D}, 6 \mathrm{C}$ \\
\hline 6 & male & $<10$ & ND & $1 \mathrm{~A}, 1 \mathrm{C}, 3 \mathrm{~B}, 3 \mathrm{D}, 3 \mathrm{E}, 3 \mathrm{~F}$ \\
\hline 7 & female & 10 & quadriceps & $1 \mathrm{~A}$ \\
\hline 8 & male & $<10$ & ND & 1B \\
\hline 9 & male & 8 & abdominal wall & 1B \\
\hline 10 & male & 19 & paravertebral & $6 \mathrm{~B}$ \\
\hline 11 & female & 17 & paravertebral & $2 \mathrm{E}, 2 \mathrm{~F}, 6 \mathrm{~B}, 6 \mathrm{C}$ \\
\hline \multicolumn{5}{|c|}{ healthy and aged donors } \\
\hline 12 & male & 48 & ND & $2 \mathrm{~A}$ \\
\hline 13 & male & $>30$ & ND & $2 \mathrm{~A}, 2 \mathrm{~B}$ \\
\hline 14 & female & 43 & ND & 2A, 2B \\
\hline 15 & male & 51 & ND & $2 \mathrm{~A}, 2 \mathrm{~B}, 5 \mathrm{~B}$ \\
\hline 16 & male & 68 & ND & 5D, 5B \\
\hline 17 & male & 29 & ND & $5 \mathrm{D}, 5 \mathrm{~B}$ \\
\hline 18 & male & 34 & ND & 5B \\
\hline \multicolumn{5}{|c|}{ DMD donors } \\
\hline 19 & male & 16 & paravertebral & $2 \mathrm{C}, 2 \mathrm{E}, 6 \mathrm{~A}, 2 \mathrm{~F}$ \\
\hline 20 & male & 15 & paravertebral & $2 \mathrm{C}, 6 \mathrm{~A}, 2 \mathrm{~F}$ \\
\hline 21 & male & 16 & paravertebral & $2 \mathrm{E}, 2 \mathrm{D}, 6 \mathrm{~A}, 6 \mathrm{~B}, 6 \mathrm{C}, 2 \mathrm{~F}$ \\
\hline 22 & male & 16 & gluteus maximus & 2C, 2E, 6A, 6B, 6C \\
\hline 23 & male & 15 & paravertebral & 2C, $2 \mathrm{D}$ \\
\hline 24 & male & 11 & tensor fasciae latae & $2 \mathrm{C}, 2 \mathrm{D}, 6 \mathrm{~A}, 6 \mathrm{~B}, 6 \mathrm{C}$ \\
\hline 25 & male & 14 & paravertebral & $2 \mathrm{D}$ \\
\hline 26 & male & 15 & deltoid & $2 \mathrm{C}, 2 \mathrm{E}, 6 \mathrm{~A}$ \\
\hline
\end{tabular}

\section{Skeletal muscle progenitor isolation}

Tissue samples were obtained as res nullius from surgeries or diagnostic biopsies performed in local hospitals. All protocols for healthy young donors aged from 1 to 19 years and for healthy aged donors from 29 to 68 years were approved by the Centre Hospitalier Universitaire de Nice Review Board, according to the rules of the French Regulatory Health Authorities and with the informed consent of the parents for young donors. Samples were placed in F10 medium and transferred to the laboratory. DMD frozen samples from donors aged 11 to 16 years were provided by the Myobank of the Institut de Myologie, AFM-Téléthon. Characteristics of healthy and DMD muscles are reported in Table 1. Skeletal muscle cells were isolated by a standard method $[14,28]$. Briefly, healthy or DMD skeletal muscles were minced into $1 \mathrm{~mm}^{3}$ fragments and digested at $37^{\circ} \mathrm{C}$, first using Liberase (Roche Diagnostics, Meylan, France) for 1 hour and then using $0.25 \%$ trypsin-EDTA for 20 minutes. The enzymatic reaction was stopped by adding $10 \%$ fetal bovine serum. The suspension was homogenized, and finally cells were pelleted by centrifugation and platted in growth culture medium.

\section{Human skeletal muscle FAP and MP isolation}

FAPs and MPs were isolated by a standard method $[13,14]$. Adherent cells derived from the digestion and amplification of healthy and DMD skeletal muscle biopsies were sorted by flow cytometry with the BD FACSARIA II sorter equipped with 4 lasers and the FACSDiva software (Becton, Dickinson and Company, Franklin Lakes, USA). Briefly, about 2000000 cells were labelled with anti-CD56-APC and CD140a-PE (PDGFR $\alpha$ ) antibodies or the relevant isotype control in PBS 1X, EDTA 2mM and 10\% FBS for 30 minutes at $4{ }^{\circ} \mathrm{C}$. The labelled-cell suspension was filtered through $40-\mu \mathrm{m}$ cell strainers, pelleted by centrifugation and re-suspended in PBS/EDTA/Fetal bovine serum for isolation by the flow cytometer. For CD140a-PE, fluorescence was excited with the 561-nm laser and measured with a 586/15 bandpass filter. For CD56APC, fluorescence was excited with the 633-nm laser and measured with a 670/14 bandpass filter. Unlike 


\section{Cellular Physiology Cell Physiol Biochem 2019;53:1029-1045 \begin{tabular}{l|l|l} 
and Biol: $10.33594 / 000000196$ & 2019 The Author(s). Published by
\end{tabular} and BIOChemistry Published online: 23 December 2019 Cell Physiol Biochem Press GmbH\&Co. KG \\ Moratal et al.: Myoblast Effects on Fibro-Adipogenic Progenitors}

MPs, FAPs are negative for myogenic marker CD56 and positive for PDGFR $\alpha$ (Supplementary Fig. 1A - for all supplemental material see www.cellphysiolbiochem.com).

\section{Growth and differentiation of FAPs and MPS}

The growth culture medium was Ham's F10 medium supplemented with 20\% FBS, 10 mM Hepes, $10^{-6} \mathrm{M}$ dexamethasone, $2.5 \mathrm{ng} / \mathrm{ml}$ basic fibroblast growth factor (FGF2), $100 \mathrm{U} / \mathrm{ml}$ penicillin, and $100 \mathrm{mg} /$ $\mathrm{ml}$ streptomycin. Myogenic and adipogenic differentiations were induced by switching to differentiation medium (DM): Ham's F10/F12/low-glucose DMEM with 2mM glutamine (2v/1v/1v) supplemented with 1\% horse serum (Gibco, Thermo Fisher Scientific, Villebon sur Yvette, France), $500 \mathrm{nM}$ dexamethasone, $50 \mu \mathrm{M}$ 1-methyl-3-isobutylmethyl-xanthine (MIX), $7.5 \mu \mathrm{g} / \mathrm{ml}$ insulin, $7.5 \mu \mathrm{g} / \mathrm{ml}$ transferrin, $0.1 \mathrm{nM}$ triiodothyronine, and $50 \mathrm{nM}$ rosiglitazone (PPAR $\gamma$ agonist). Three days later, cells were placed in the same medium lacking MIX and dexamethasone. This differentiation medium was replaced every 3 days and cells were collected after 10 days of differentiation to analyze fibrogenesis and adipogenesis, or 7 days for myogenesis. Fibrogenic differentiation was obtained with the above DM complemented with 5ng/ml TGF $\beta 1$ for 5 days.

\section{Validation of FAP and MP differentiation}

In the dual myogenic-adipogenic culture medium, FAPs differentiated only into adipocytes containing lipid droplets and MPs only into multinucleated myotubes (Supplementary Fig. 1B). Accordingly, the adipogenic markers fatty acid binding protein 4 (FABP4), Adipsin and perilipin 1 (PLIN1) were specifically expressed in FAP differentiating cultures and the myogenic markers muscle creatine kinase $(M C K)$, myogenin $(M Y O G)$ and $\delta$-sarcoglycan $(S G C D)$ were specifically expressed in MP differentiating cultures (Supplementary Fig. 1D). In a fibrogenic culture medium, only FAPs differentiated into alpha smooth muscle actin ( $\alpha \mathrm{SMA}$ )-positive myofibroblasts with stress fibers (Supplementary Fig. 1C) and the TGF $\beta 1$ induction of the fibrogenic markers collagen type I alpha 1 chain (COL1A1), fibronectin 1 (FN1) and aSMA was clearly stronger in FAPs than MPs (Supplementary Fig. 1E). In the next experiments, MP interactions on FAPs were assessed with FAPs and MPs extracted from the same human donor and a collection of donors was used.

For co-cultures experiments, 100000 FAPs and 100000 MPs were seeded in a well of a 12-well plate. One day later the growth culture medium was replaced by the differentiation medium. Data were compared to mono-cultures conducted in the same conditions.

\section{Production of conditioned-media}

To generate conditioned-medium from proliferating-MPs (MP CM) used in proliferation experiments, $\mathrm{CD}^{\circ} 6^{+}$cells were cultured in the growth medium and at 60-80\% of confluence treated with fresh growth medium for 1 day. To generate conditioned-medium from undifferentiated MPs (MP CM) used in differentiation experiments, $\mathrm{CD}_{5} 6^{+}$cells were cultured in the growth medium and at $80 \%$ of confluence treated with cytokines-free differentiation medium containing $1 \%$ horse serum for 1 day. To generate conditioned-medium from differentiated myotubes (Myotube CM) used for differentiation experiments, confluent $\mathrm{CD}^{2} 6^{+}$cells were differentiated with the differentiation medium and at day 6 treated with cytokines-free differentiation medium for 1 day. At the same time, control CM (Ctrl CM) was obtained by incubating growth or differentiation medium without cells in the incubator and collected 24 hours later. After collecting, all these conditioned-media were spined at $2000 \mathrm{rpm}$ for 10 minutes, then stocked at $-20^{\circ} \mathrm{C}$. They were used without dilution. Dexamethasone and FGF2 were added before freezing in all conditionedmedia employed in proliferation experiments. The cocktail of myogenic-adipogenic cytokines was added before freezing in all conditioned-media employed in differentiation experiments.

\section{MTT assays}

Cells were seeded at 1500 cells/well in a 96-well plate in the presence of adequate conditionedmedium. Cellular viability was measured by adding $10 \mu \mathrm{l}$ of $5 \mathrm{mg} / \mathrm{ml}$ thiazolyl blue tetrazolium bromide (MTT, Sigma-Aldrich) in wells and the plate was incubated at $37^{\circ} \mathrm{C}$ for 4 hours in culture hood. Then, the medium was removed and MTT crystals were dissolved in $150 \mu \mathrm{l}$ of DMSO/isopropanol (v/v). The plate was incubated at room temperature for 10 minutes with gently agitation, and absorbance was read at $570 \mathrm{~nm}$, and $620 \mathrm{~nm}$ which is the reference filter. Each condition was done in triplicate. 


\section{Cellular Physiology Cell Physiol Biochem 2019;53:1029-1045 \\ \begin{tabular}{l|l|l}
\hline DOI: 10.33594/000000196 & (c) 2019 The Author(s). Published by
\end{tabular} \\ \begin{tabular}{l|l} 
Published online: 23 December 2019 & Cell Physiol Biochem Press GmbH\&Co. KG \\
\hline
\end{tabular} \\ Moratal et al.: Myoblast Effects on Fibro-Adipogenic Progenitors}

\section{Immunofluorescence analysis}

Cells plated on slides were fixed with Histofix 4\% (Carl Roth, Lauterbourg, France) for 10 minutes, permeabilized and saturated with $0.1 \%$ Triton X-100 / 3\% bovine serum albumin for 30 minutes at room temperature, and sequentially incubated with primary antibody (Sigma-Aldrich) overnight at $4^{\circ} \mathrm{C}$ then with secondary antibody for 45 minutes at room temperature. PBS wash was performed three times between all steps. Cells were finally mounted in Mowiol containing DAPI and visualized with an Axiovert microscope (Carl Zeiss, Le Pecq, France) under oil immersion and pictures were captured and treated with AxioVision software (Carl Zeiss).

\section{RNA extraction and Reverse Transcription quantitative Polymerase Chain Reaction}

Total RNA was extracted using TRI-reagent (Euromedex, Souffelweyersheim, France). The protocols of RNA extraction and quantitative RT-PCR were previously mentioned [13]. The housekipping gene TATA box-binding protein (TBP) was used as reference. The 5'-3' sequences of forward and reverse primers were, respectively,

ACGCCAGCTTCGGAGAGTTC and CAAACCGCTTGGGATTATATTCG for TBP,
ATGGGATGGAAAATCAACCA and TGCTTGCTAAATCAGGGAAAA for FABP4,
AGGGTCACCCAAGCAACAAAG and TACGTGGCCCATGCTGATCT for adipsin,
ACCATCTCCACCCGCCTC and GATGGGAACGCTGATGCTGT for PLIN1,
ACCTGCGTGTACCCCACTCA and CCGCCATACTCGAACTGGAA for COL1A1,
CTGGCCGAAAATACATTGTAAA and CCACAGTCGGGTCAGGAG for FN1,
TGCCTGCATGGGCAAGTGA and CTGGGCAGCGGAAACG for $\alpha S M A$,
CTGCGTGGCTGGTGATGAG and CAGGTCGTCTCCACCCTTGA for $M C K$,
CAGCTCCCTCAACCAGGAG and GCTGTGAGAGCTGCATTCG for $M Y O G$,
CGGGAGTGTTGAGTGAAGGG and CTCCGGTGGTGAGTGTACTG for $S G C D$,
TGCAAAGCCTTCAGCAATG and TTTTCGCAGCGAGCTAGGAT for $G L I 1$.

\section{Immunoblot analysis}

Cells were lysed in RIPA buffer consisting of $50 \mathrm{mM}$ Tris- $\mathrm{HCl} \mathrm{pH}$ 8.0, $150 \mathrm{mM} \mathrm{NaCl}, 0.1 \%$ SDS, $0.5 \%$ sodium deoxycholate, $5 \mathrm{mM} \mathrm{NaF}, 2.5 \mathrm{mM} \mathrm{NA}_{4} \mathrm{P}_{2} \mathrm{O}_{7}, 1 \% \mathrm{NP} 40,2 \mathrm{mM}$ sodium vanadate and protease inhibitor cocktail (Roche Diagnostics, Meylan, France). The protein content was determined according the BCA method (Pierce BCA Protein Assay Kit, Thermoscientific, Illkirch Graffenstaden, France \#23227). Cell lysates were centrifuged at $13000 \mathrm{~g}$ for 10 minutes at $4^{\circ} \mathrm{C}$ and the supernatants were recovered.

$10 \mu \mathrm{g}$ of proteins were resolved by $7.5 \%$ SDS-PAGE under reducing conditions and transferred to Immobilon-P membranes (Millipore Corporation, Bedford, USA). The membranes were probed with the bound primary antibody that was detected by horseradish peroxidase-conjugated secondary antibody (Promega, Charbonnières les Bains, France) and visualized with an electrochemical luminescence detection kit (Bio-Rad, Marnes la Coquette, France). The band intensity was measured using the Quantity One software (Bio-Rad).

\section{Statistical analysis}

Differences between data groups were evaluated for significance using the two-tailed unpaired Student's t-test. A P-value $<0.05$ was considered significant. The data are presented as mean \pm S.E. of the mean of at least three independent muscle biopsies in duplicate or triplicate. Muscle biopsy samples were randomly chosen in our collection.

\section{Results}

\section{MPs stimulate FAP proliferation via the PI3K/Akt signaling pathway}

The proliferation of FAPs was found to be accelerated in the presence of MP conditioned medium (MP CM); this effect was soon as 24 hours after the addition of conditioned medium and was observed for two more days (Fig. 1A). FAP-induced proliferation was associated with a two-fold increase of Akt phosphorylation (Fig. 1B). Addition of the phosphoinositide 3-kinase (PI3Kinase) inhibitor wortmannin alleviated the pro-proliferative action of MP 
conditioned medium on FAPs (Fig. 1C) without induction of cell mortality (data not shown). No significant variation of extracellular signal-regulated kinases 1/2 (ERK1/2) phosphorylation, another important regulation pathway of cell proliferation, was observed in the same conditions (Supplementary Fig. 2). Taken together, these results show that MPsecreted factors can stimulate FAP proliferation via an over-activation of PI3K/Akt pathway.

The regulation of FAP proliferation by $M P s$ is altered with aging and DMD

We checked whether the regulation of FAP proliferation by MPs was modified with aging and dystrophic environment. Unlike young progenitors (Fig. 1A), no stimulation of aged FAP proliferation by conditioned medium from aged MPs was observed (Fig. 2A). Consistently, this was not associated with a stimulation of Akt phosphorylation (Fig. 2B). To note, the proliferation capacity of aged FAPs was similar to young FAPs (Supplementary Fig. 3A). These results indicate that MPs do not stimulate PI3Kinase/Akt pathway resulting in the absence of stimulation of FAP proliferation for progenitors from aged donors.

As with aged progenitors, DMD MPs did not have a stimulatory effect on DMD FAP proliferation, in conditioned medium experiments (Fig. 2C). Surprisingly, the significant increase of Akt phosphorylation was maintained in DMD FAPs cultured with conditioned medium from DMD MPs (Fig. 2D), as observed with healthy progenitors (Fig. 1B). Interestingly, the stimulatory effect of DMD FAP proliferation was rescued with healthy MPs (Fig. 2E), while DMD MPs did not increase the proliferation of healthy FAPs (Fig. 2F). Moreover, DMD FAP proliferation rate was similar to healthy FAPs (Supplementary Fig. 3B). Thus, we can conclude that factors secreted by MPs are modified in a dystrophic environment resulting in alteration of FAP proliferation regulation.

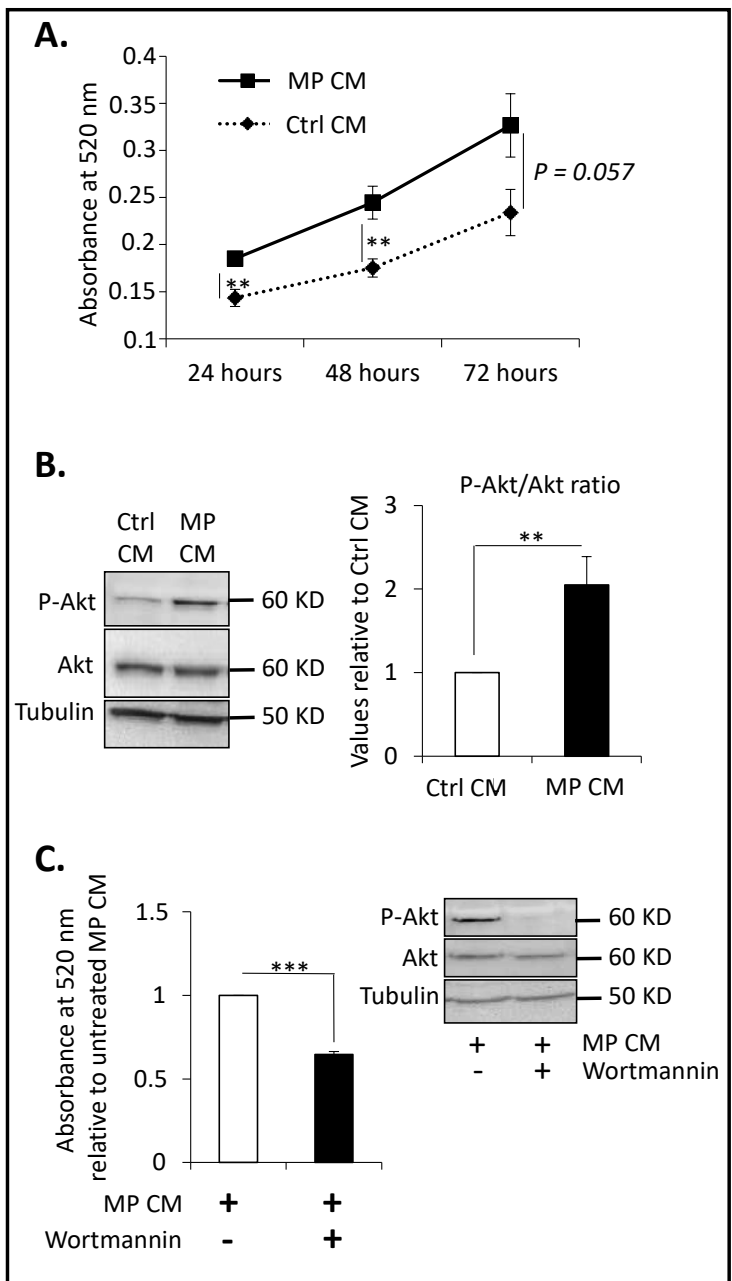

Fig. 1. The MP-dependent stimulation of FAP proliferation is mediated by Akt signaling. FAPs proliferated in the presence of conditioned medium (CM) from MPs (MP CM) compared to control conditioned medium (Ctrl CM). (A) Proliferation of FAPs was measured by MTT assays 24 hours, 48 hours and 72 hours after plating ( $n=5$ donors). (B) Confluent FAPs were treated with Ctrl CM or MP CM and total proteins were extracted 15 minutes later. Protein expression of phosphorylated Akt (P-Akt), total Akt (Akt) and tubulin was assessed by western blot (left panel). P-Akt band intensity was quantified and normalized to total Akt signals (right panel) ( $n=3$ donors). (C) FAPs proliferated in the presence of MP $\mathrm{CM}$ with or without wortmannin. Proliferation of FAPs was measured by MTT assays 48 hours after plating. Protein levels of P-Akt, total Akt and tubulin was assessed by western-blot. Representative immunoblot is shown ( $\mathrm{n}=3$ donors). ${ }^{* * *} \mathrm{P}<0.001$; ${ }^{* *} \mathrm{P}<0.01$; $\mathrm{p}$ values close to significance are indicated. 
Fig. 2. The MP-dependent regulation of FAP proliferation is altered in aging and DMD progenitors. (A and B) Aged FAPs proliferated with control conditioned medium (Ctrl CM) or conditioned medium from aged MPs (Aged MP CM). (A) Proliferation was measured by MTT assays 24 hours, 48 hours and 72 hours after plating. $(n=4$ aged donors). (B) 15 minutes after addition of the conditioned medium, total proteins were extracted; expression levels of phosphorylated Akt, total Akt and tubulin were assessed by western blot. Representative immunoblot is shown ( $\mathrm{n}=3$ aged donors). ( $\mathrm{C}$ and D) DMD FAPs proliferated with control conditioned medium (Ctrl CM) or conditioned medium from DMD MPs (DMD MPs CM). (C) Proliferation was measured by MTT assays 24 hours, 48 hours and 72 hours after plating. (n=6 DMD donors). (D) 15 minutes after addition of the conditioned medium, total proteins were extracted; expression levels of phosphorylated Akt, total Akt and tubulin were assessed by western-blot. Representative immunoblot is shown ( $n=3$ DMD donors). (E) DMD FAPs proliferated with control conditioned medium (Ctrl CM) or conditioned medium from Healthy MPs (Healthy MP CM). Proliferation was measured by MTT assays 24 hours, 48 hours and 72 hours after plating. ( $n=3$ DMD and $n=1$ healthy donor). (F) Healthy FAPs proliferated with control conditioned medium (Ctrl CM) or conditioned medium from DMD MPs (DMD MP CM). Proliferation was measured by MTT assays 24 hours, 48 hours and 72 hours after plating ( $\mathrm{n}=3 \mathrm{DMD}$ and $\mathrm{n}=3$ healthy donors). $\mathrm{ns}=$ non-significant. ${ }^{*} \mathrm{P}<0.05$.

Molecules secreted by myotubes inhibit adipogenesis and stimulate fibrogenesis of FAPs

Adipogenic and fibrogenic potentials of FAPs derived from healthy young donors were investigated in FAP/MP co-cultures with direct cell contact. In these conditions, FAP adipogenesis was abolished as indicated by the decreased number of mature adipocytes (Fig. 3A) and inhibition of mRNA expression of the specific adipogenic markers fatty acid binding protein 4 (FABP4) and perilipin 1 (PLIN1) (Fig. 3B). Conversely, FAP fibrogenesis was clearly increased. Alpha smooth muscle actin $(\alpha \mathrm{SMA})$-positive myofibroblasts appeared in FAP/MP co-cultures (Fig. 3C) and expression of the specific fibrogenic mRNAs collagen type I alpha 1 chain (COL1A1) and fibronectin 1 (FN1) was noticeably higher in co-cultures than in FAP and MP mono-cultures (Fig. 3D).

Under the dual myogenic-adipogenic culture conditions, FAP/MP co-cultures rapidly contained a mix of MPs and myotubes. To define their respective roles, conditioned media from MPs (MP CM) or from differentiated MPs (Myotube CM) were added to FAP monocultures. Expression of adipogenic markers in FAP cultures was significantly decreased by conditioned medium from myotubes but not by conditioned medium from MPs (Fig. 3E). Similarly, only conditioned medium from myotubes and not from MPs stimulated significant expression of fibrogenic markers in FAPs (Fig. 3F).

These data show that myotubes secrete molecules that regulate the FAP fate by inhibiting adipogenesis and stimulating fibrogenesis. 


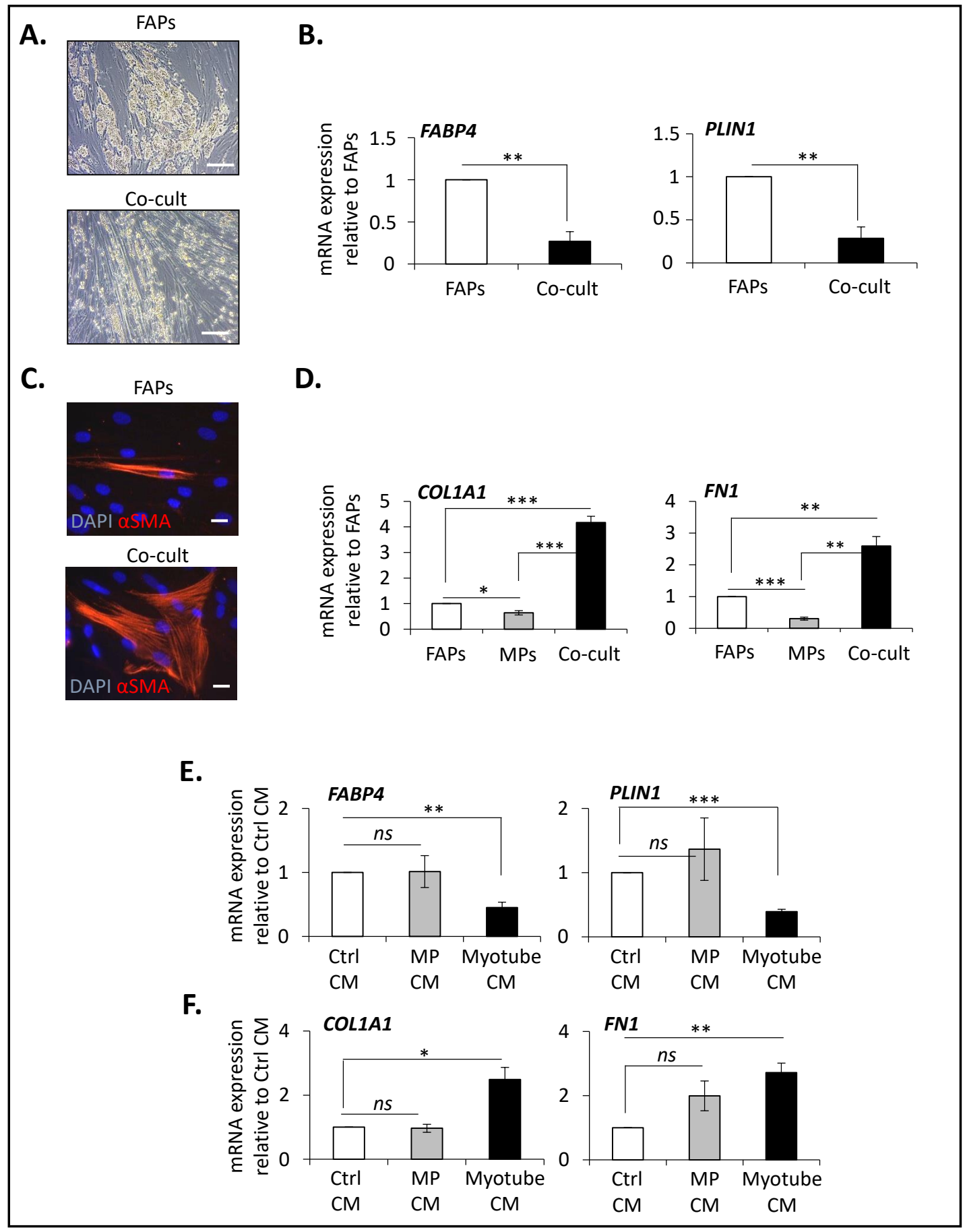

Fig. 3. Myotubes inhibit adipogenesis and stimulate fibrogenesis of FAPs. (A to D) For co-culture assays, FAPs were cultured alone (FAPs) or with MPs (Co-cult) for 10 days in differentiation medium. (A) FAPderived adipocytes were visualized with phase-contrast microscopy (scale bar: $20 \mu \mathrm{m}$ ). (C) FAP-derived myofibroblasts were visualized by $\alpha$ SMA immuno-labelling. Nuclei were labelled with DAPI (scale bar: $5 \mu \mathrm{m}$ ). Expression of the adipogenic genes FABP4 and PLIN1 (B) and expression of the fibrogenic genes COL1A1 and FN1 (D) were measured by quantitative Q-PCR ( $\mathrm{n}=6$ donors). (E and F) For conditioned medium experiments, FAPs were cultured in the presence of conditioned medium from control (Ctrl CM), MPs (MP CM) or differentiated myotubes (Myotube CM) for 10 days. Expression of adipogenic genes (E) and fibrogenic genes (F) was measured by quantitative Q-PCR ( $\mathrm{n}=4$ donors). ${ }^{* * *} \mathrm{P}<0.001$; $^{* *} \mathrm{P}<0.01$; $^{*} \mathrm{P}<0.05$; ns= non-significant. 
Myotubes regulate FAP differentiation via stimulation of the ALK/Smad2 signaling pathway

We recently showed that adipogenesis is negatively regulated in FAPs at the expense of fibrogenesis by factors from the TGF $\beta$ family [14]. We wondered whether this pathway is implicated in the regulation of FAP fibro-adipogenesis by myotubes from healthy young donors.

SB431542 is a specific inhibitor of TGF $\beta$ superfamily type I activin receptor-like kinase (ALK) receptors ALK4, ALK5, and ALK7. Remarkably, the expression of adipogenic markers was increased and the expression of fibrogenic markers was even decreased (Fig. 4A) in cocultures treated with SB431542 compared to untreated co-cultures.

As expected, we found that phosphorylation of Smad2, a downstream effector of ALK activation, was activated in FAPs cultured with myotube conditioned medium (Fig. 4B).

Fig. 4. Regulation of FAP fate by myotubes requires activation of A L K / S m a d 2 pathway and GLI1 expression in FAPs. (A) FAPs were cultured with MPs (Cocult) for 10 days in differentiation medium with or without SB431542 that is a specific inhibitor of ALK4, ALK5, and ALK7. Expression of FABP4, PLIN1, COL1A1 and FN1 was measured by quantitative Q-PCR ( $n=3$ donors). (B) FAPs were cultured with Ctrl $\mathrm{CM}$ or myotube $\mathrm{CM}$ for 1 hour, then total proteins were extracted. Expression levels of phosphorylated Smad2 (P-Smad2), total Smad2 and tubulin were assessed

A.
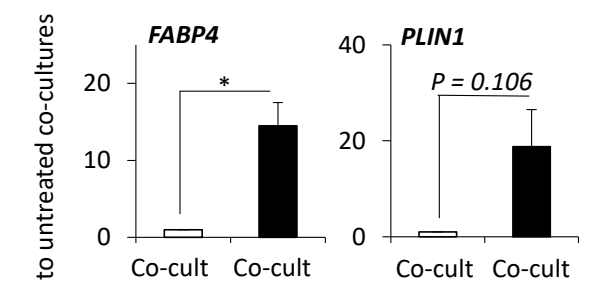

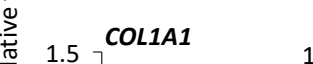
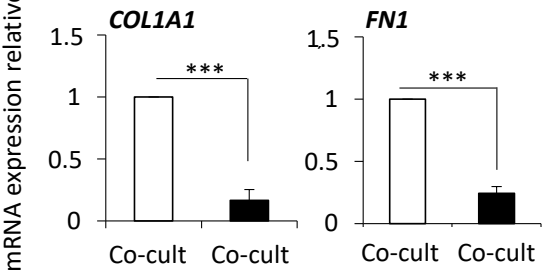

SB431542

C.
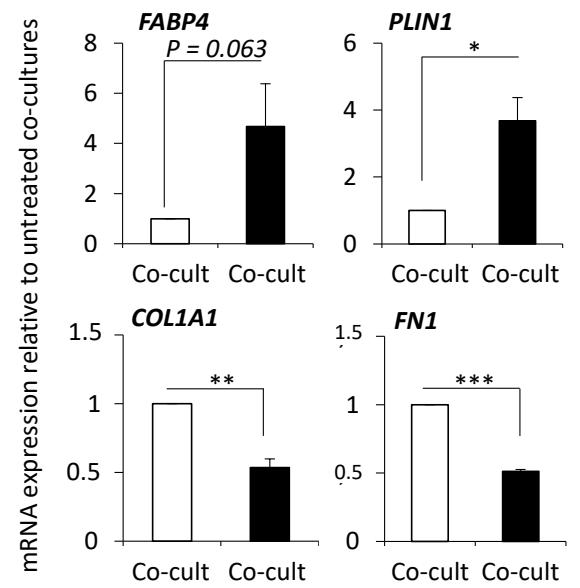

Cyclopamine $\quad-\quad+$
B.

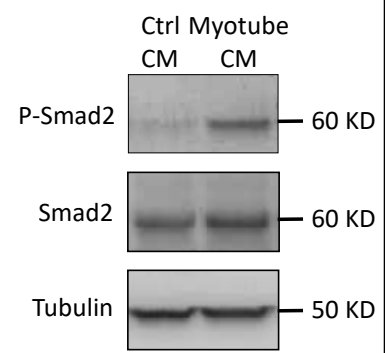

D.

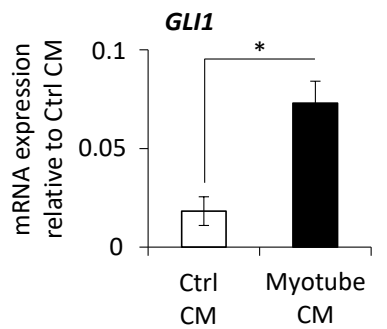

by western-blot. Representative immunoblot is shown ( $\mathrm{n}=3$ donors). (C) FAPs were cultured with MPs (Co-cult) for 10 days in differentiation medium with or without cyclopamine that is a specific inhibitor of GLI1 expression. Expression of FABP4, PLIN1, COL1A1 and FN1 was measured by quantitative Q-PCR ( $n=3$ donors). (D) FAPs were cultured with Ctrl CM or myotube CM for 10 days in differentiation medium. Expression of GLI1 was measured by quantitative Q-PCR ( $\mathrm{n}=3$ donors). ${ }^{* * *} \mathrm{P}<0.001 ;{ }^{* *} \mathrm{P}<0.01 ;{ }^{*} \mathrm{P}<0.05$; $\mathrm{p}$ values close to significance are indicated. 
Therefore, ALK/Smad2 signaling pathway within FAPs is an essential mediator of the regulation of FAP adipogenic and fibrogenic differentiations by MP-derived myotubes.

\section{Myotubes regulate FAP differentiation via the stimulation of GLI1 expression}

GLI1 is a downstream effector of Hedgehog signaling that plays a crucial role in the adipogenesis of mesenchymal stem cells [29] and in the regulation of intramuscular fibrosis in response to injury [30]. We assessed the importance of this signaling in the control of FAP fibro-adipogenesis by myotubes in progenitors derived from healthy young donors.

Cyclopamine is an inhibitor upstream of the Hedgehog pathway by directly inhibiting the Smoothened receptor that induces a drastic inhibition of GLI1 expression. Cyclopamine had no effect on autonomous adipogenesis and fibrogenesis of FAPs (data not shown). Interestingly, cyclopamine alleviated the inhibition of adipogenesis and reduced the stimulation of fibrogenesis in FAP/MP co-cultures, with an increase of expression of the adipogenic markers FABP4 and PLIN1 and a decrease expression of fibrogenic markers COL1A1 and FN1 (Fig. 4C). Consistent with this, GLI1 expression was increased in FAPs cultured with myotube conditioned medium (Fig. 4D).

These results indicate that GLI1 expression is essential for the regulation of FAP differentiations by myotubes for healthy young progenitors.

The regulation of FAP differentiation by myotubes is altered with aging and DMD

Firstly, we found that differentiation potentials of aged FAPs were higher than young FAPs as shown by the high decrease in expression of FABP4 (Supplementary Fig. 3C) and COL1A1 (Supplementary Fig. 3D) in aged FAPs in comparison to young progenitors. Secondly, we inspected whether aging or DMD could interfere with the myotube-dependent control of FAP differentiation. Unlike young progenitors (Fig. 3A and B), FAP adipogenesis was not inhibited in aged co-cultures. Neither phenotype modification (Fig. 5A) nor decrease in FABP4 expression were observed (Fig. 5B). Regarding the fibrogenic differentiation, specific marker (COL1A1 and FN1) expressions were higher in aged co-cultures than in aged FAP mono-cultures, but this might be due to the high expression of fibrogenic mRNAs by aged MPs (Fig. 5B). To get more insights into the effects of aging, young MPs instead of aged MPs, were co-cultured with aged FAPs. The inhibition of aged FAP adipogenesis was restored according to the important reduction of adipocyte amount (Fig. 5C) and the decrease of FABP4 expression (Fig. 5D). In parallel, a strong rise of fibrogenesis was obtained, as seen with the increased expression of COL1A1 and FN1 concomitantly with a low level of expression in both FAP and MP mono-cultures (Fig. 5D). These results show that aging causes an alteration in the intrinsic fibro-adipogenic potential of aged FAPs and in control of FAP differentiation by the myogenic lineage.

As with healthy progenitors (Fig. 3A and B) and in contrast with aged progenitors (Fig. 5A and 5B), DMD MPs inhibited DMD FAP adipogenesis. This was observed in DMD FAP/MP cocultures, with the decrease of FABP4 expression (Fig. 6A), as well as in cultures of DMD FAPs submitted to conditioned medium from DMD myotubes with the high decrease expression of PLIN1 (Supplementary Fig. 4A). Unlike healthy progenitors (Fig. 4C and D), DMD MPs did not significantly stimulate DMD FAP fibrogenesis. As shown in Fig. 6A, expression of COL1A1 and FN1 was not significantly increased in co-cultures; expression of FN1 was higher in co-cultures than in DMD FAP mono-cultures but were not significantly different from the expression in DMD MP mono-cultures. Accordingly, DMD myotube conditioned medium did not stimulate COL1A1 expression in FAPs (Supplementary Fig. 4A). To further characterize the impacts of DMD on regulation of FAP differentiation, DMD MPs were replaced by healthy age-matching MPs. The inhibition of DMD FAP adipogenesis was maintained as shown by the high decrease in expression of FABP4 in DMD FAP/healthy MP co-cultures (Fig. 6B). Unlike results obtained with DMD MPs, DMD FAP fibrogenesis was stimulated in co-cultures with healthy MPs. The stimulation of COL1A1 and FN1 expressions was two-times higher in DMD FAP/healthy MP co-cultures (Fig. 6B) than in DMD FAP/DMD MP co-cultures (Fig. 6A). Consistently, an increase in the COL1A1 expression was similarly found in DMD FAPs 


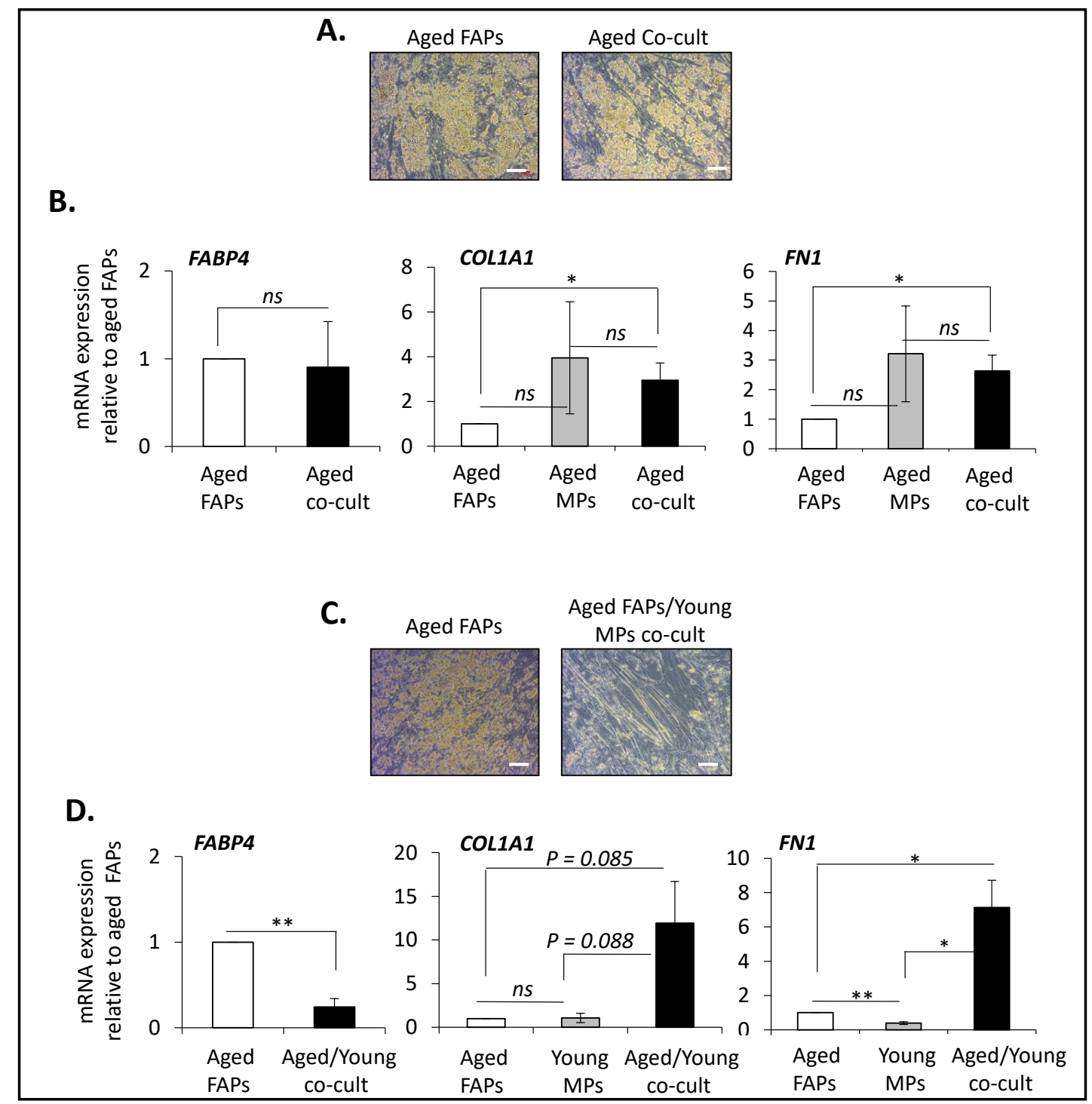

Fig. 5. The myotube-dependent regulation of FAP differentiation is altered in aging. Aged FAPs were cultured alone or with aged MPs (Aged co-cult) (A and B) or with Young MPs (Aged/Young co-cult) (C and D) for 10 days in differentiation medium. (A and C) Adipocytes derived from aged FAPs were visualized by phasecontrast microscopy (scale bar: $5 \mu \mathrm{m}$ ). (B and D) Expression of FABP4, COL1A1 and FN1 was measured by quantitative $\mathrm{Q}-\mathrm{PCR}\left(\mathrm{n}=4\right.$ aged donors and $\mathrm{n}=3$ young donors). ${ }^{* *} \mathrm{P}<0.01 ;{ }^{*} \mathrm{P}<0.05$; ns= non-significant; $\mathrm{p}$ values close to significance are indicated.

differentiated in healthy myotube conditioned medium (Supplementary Fig. 4B). On the other hand, we assessed the effects of DMD MPs on differentiation of healthy FAPs in coculture (Fig. 6C). Adipogenesis was not decreased as shown by the similar expression of FABP4 in co-cultures and healthy FAP mono-culture. Fibrogenesis was not stimulated in cocultures since COL1A1 expression was not significantly different from healthy FAP monoculture and FN1 expression was similar to DMD MP mono-culture. To note, FAP differentiation into adipocytes [14] and myofibroblasts (Supplementary Fig. 3D) was conserved in FAPs isolated from skeletal muscles of DMD patients. Surprisingly, these results indicate that the control of FAP fibrogenesis by myotubes is abolished while the inhibition of adipogenesis is conserved in DMD progenitors. Interestingly, the deficiency in the regulation of DMD FAP fibrogenesis implicates an alteration of myotubes-secreted factors induced by the dystrophic environment. 


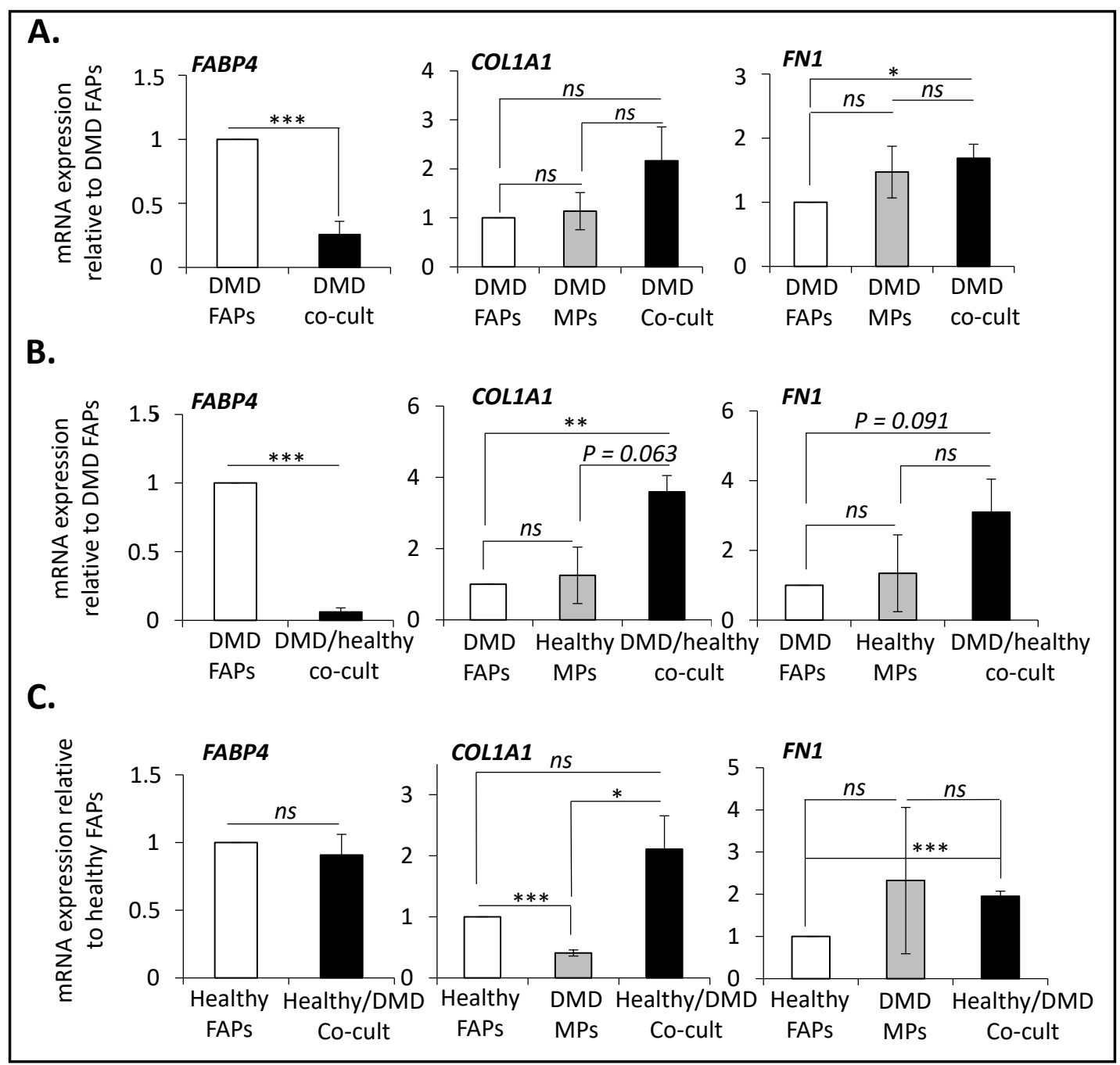

Fig. 6. The myotube-dependent regulation of FAP differentiation is altered in DMD. DMD FAPs were cultured alone or with DMD MPs (DMD co-cult) (A) or with Healthy MPs (DMD/Healthy co-cult) (B) for 10 days in differentiation medium. (C) Healthy FAPs were cultured alone or with DMD MPs (Healthy/DMD co-cult) for 10 days in differentiation medium. Expression of FABP4, COL1A1 and FN1 was measured by quantitative $\mathrm{Q}$-PCR ( $\mathrm{n}=6$ DMD donors and $\mathrm{n}=3$ healthy donors). ${ }^{* *} \mathrm{P}<0.001$; $^{* *} \mathrm{P}<0.01{ }^{*} \mathrm{P}<0.05$; $\mathrm{ns}=$ non-significant; $\mathrm{p}$ values closed to significance are indicated.

Thus, aging and DMD have a different impact on the potential of myotube to regulate FAP differentiation.

\section{Discussion}

In response to injury, skeletal muscle undergoes a highly orchestrated degenerative and regenerative process for which satellite cells are central actors as myogenic progenitors. FAPs have recently emerged as other important actors of regeneration in healthy and dystrophic mouse muscles. In addition to cells from the immediate niche surrounding satellite cells, the interstitial cells FAPs have the potential to regulate satellite cell fate and thus contribute to muscle regeneration $[3,5]$. These findings stress the importance of studying the FAP/MP interactions in humans. Here, we focused on the in vitro regulation of FAPs by MPs isolated from human muscles. 
Firstly, we showed that MPs are actors of a positive regulation of FAP proliferation mediated by the Akt phosphorylation. This is in accordance with previous data showing that activation of PDGFR $\alpha$ signaling inducing PI3K-Akt pathway stimulates proliferation of FAPs $[15,31,32]$. While platelet-derived growth factor-AA could be a likely factor controlling FAP proliferation [15, 32], further investigations are needed to identify factors activating PI3KAkt pathway in FAPs submitted to MP secretome.

Another incidence of MP lineage on FAPs is the regulation of FAP differentiation. Myotubes, but not MPs, inhibited adipogenesis of FAPs as reported in mice [8]. Another finding is the profibrogenic role of human myotubes on FAPs, which has not been documented in mice. This other function of myotubes is of special interest and emphasis the importance of the coordination between FAPs and myotubes for muscle regeneration. Thus, myotubesecreted factors control a differentiation balance between fibrogenesis and adipogenesis of FAPs.

Mechanistically, we showed that myotube-secreted factors highly phosphorylated Smad2 in FAPs, and this phosphorylation is mediated by ALK4/5/7 receptor activity. Among canonical members of the TGF $\beta$ superfamily, TGF- $\beta 1$ and activin A are known to exert antiadipogenic [33-35] and pro-fibrogenic effects [36, 37], through Smad2 pathway activation [38]. In accordance, we previously found that TGF $\beta 1$ and activin A inhibit adipogenesis in FAPs [13]. We have tested the involvement of these two factors in FAP regulations by myotubes. We disproved this hypothesis since the immuno-blocking of TGF $\beta$ and/or activin A did not counteract the effects of myotube conditioned media on FAPs fibro-adipogenesis (data not shown). Furthermore, no secretion of TGF $\beta 1$ by myogenic lineage was detected by Elisa assay. Members of the TGF $\beta$ family responsible to the effects on FAP differentiation remains to be identified.

In addition to ALK/Smad2 signaling, we found that the transcription factor GLI1, a final transcriptional activator of Hedgehog signaling $[39,40]$, is a central factor for the regulation of FAP differentiation by myotube-secreted factors. Indeed, myotubes secreted factors that promote GLI1 expression in FAPs resulting in the inhibition and the stimulation of FAP adipogenesis and fibrogenesis respectively. This prominence of GLI1 is in agreement with recently published results showing that activating Hedgehog signaling in FAPs blocked injury-induced intramuscular adipogenesis [26]. Interestingly, GLI1 was also reported to be an effector of functional Smad pathway $[41,42]$. This indicates a potential cooperation between ALK/Smad2 pathway and GLI1 expression in the regulation of FAP fate by myotubes, as already proposed in cancer [43].

As the intrinsic differentiation potential of FAPs, FAP regulation by MP lineage is altered with aging. MPs did not stimulate Akt phosphorylation nor consequently FAP proliferation anymore, and myotubes stopped to regulate the fibro-adipogenic potential of FAPs. This was consistent with recent results indicating that aging disturbed the myogenic support of FAPs to MPs [44]. We showed that the aging of MPs is responsible for this lack of regulation. Such results are in accordance with the functional decline of satellite cells described in sarcopenic muscles $[45,46]$. Further experiments are necessary to demonstrate that aged MPs could be incompetent to stimulate fibrogenesis and inhibit adipogenesis of young FAPs. The age effect we observed fits the accumulation of adipose tissue in aged muscles at the expenses of muscle regeneration $[47,48]$.

Similarly to aging, DMD altered the above model of FAP regulation in young donors. Interestingly, these deleterious effects can be reversed by signals from healthy MPs or myotubes suggesting that DMD altered their molecule secretion. The lack of the submembrane protein dystrophin, which is only expressed in the myogenic lineage, could lead to modifications of factors secreted by MPs and myotubes. Indirect effects to the lack of dystrophin should also be considered since the DMD MPs are submitted for years to chronic inflammatory regenerative/degenerative conditions, which have the potential to modify MP intrinsic properties. Interestingly, the anti-adipogenic effect on DMD FAPs by myotubes and the absence of positive control of DMD FAP proliferation and fibrogenesis by MPs may contribute to tentatively limit the development of intramuscular fat and fibrotic infiltrations. 
While myotubes would have a protective role against muscle adipocyte and myofibroblast accumulations, other cells that have infiltrated DMD muscles could be the source of the formation of fat and fibrotic deposits such as macrophages [14, 49].

\section{Conclusion}

In summary, we described human specific features and uncovered the role of MPs and differentiated MPs for the regulation of FAP proliferation, adipogenesis and fibrogenesis. Importantly, we also found that the incidence of FAP/MP interactions is age dependent and largely modified with DMD progenitors. Finally, we identified signaling pathways essential to mediate the regulations of FAPs by MPs and MP-derived myotubes. The identification of Hedgehog and TGF $\beta$ signaling pathways as important mediators provide molecular bases to evaluate the control of FAPs by MPs as clinical tools to improve muscle repair in healthy, aging and dystrophic muscles.

\section{Acknowledgements}

We thank JY. Kurzenne (Hôpitaux pédiatriques de Nice CHU-Lanval, Nice, France) for providing muscle biopsies from healthy donors, and Myobank-AFM Institut de Myology, Paris, France for providing muscle biopsies from Duchenne muscular dystrophy donors. The authors greatly thank Agnès Loubat from Institut de Biologie Valrose, Nice, for FAP and MP purification from human skeletal muscle biopsies.

\section{Statement of Ethics}

All protocols for healthy young donors aged from 1 to 19 years and for healthy aged donors from 29 to 68 years were approved by the Centre Hospitalier Universitaire de Nice Review Board, according to the rules of the French Regulatory Health Authorities and with the informed consent of the parents for young donors.

\section{Funding Sources}

This work was supported by the Association Française contre les Myopathies (AFM), (through grants \#15294, \#16063, and \#17708 and a PhD fellowship for CM) and the Netherland Duchene Parent Project. The French Government (National Research Agency, ANR) supported this work through the "Investments for the Future" LABEX SIGNALIFE: program reference \# ANR-11-LABX-0028-01.

\section{Author Contributions}

Christian Dani (CD), Claude A Dechesne (CAD) and Claudine Moratal (CM) designed this project. CM, Nicole Arrighi (NA) and CAD were involved in acquisition, analysis and interpretation of data. CM and CAD wrote the article. NA and CD participated to the revising of this article.

\section{Disclosure Statement}

The authors have no conflicts of interest to declare. 


\section{Cellular Physiology Cell Physiol Biochem 2019;53:1029-1045 \begin{tabular}{l|l|l} 
DOI: 10.33594/000000196 & (c)19 The Author(s). Published by
\end{tabular} and Biochemistry Published online: 23 December 2019 Cell Physiol Biochem Press GmbH\&Co. KG \\ Moratal et al.: Myoblast Effects on Fibro-Adipogenic Progenitors}

\section{References}

1 Mauro A: Satellite cell of skeletal muscle fibers. J Biophys Biochem Cytol 1961;9:493-495.

2 Pannérec A, Marazzi G, Sassoon D: Stem cells in the hood: the skeletal muscle niche. Trends Mol Med 2012;18:599-606.

3 Yin H, Price F, Rudnicki MA: Satellite cells and the muscle stem cell niche. Physiol Rev 2013;93:23-67.

4 Bentzinger CF, Wang YX, Dumont NA, Rudnicki MA: Cellular dynamics in the muscle satellite cell niche. EMBO Rep 2013;14:1062-1072.

5 Ceafalan LC, Popescu BO, Hinescu ME: Cellular players in skeletal muscle regeneration. BioMed Res Int 2014; DOI:10.1155/2014/957014.

6 Saclier M, Cuvellier S, Magnan M, Mounier R, Chazaud B: Monocyte/macrophage interactions with myogenic precursor cells during skeletal muscle regeneration. FEBS J 2013;280:4118-4130.

7 Joe AWB, Yi L, Natarajan A, Le Grand F, So L, Wang J, Rudnicki MA, Rossi FM: Muscle injury activates resident fibro/adipogenic progenitors that facilitate myogenesis. Nat Cell Biol 2010;12:153-163.

8 Uezumi A, Fukada S, Yamamoto N, Takeda S, Tsuchida K: Mesenchymal progenitors distinct from satellite cells contribute to ectopic fat cell formation in skeletal muscle. Nat Cell Biol 2010;12:143-152.

9 Vauchez K, Marolleau J-P, Schmid M, Khattar P, Chapel A, Catelain C, Lecourt S, Larghéro J, Fiszman M, Vilquin JT: Aldehyde dehydrogenase activity identifies a population of human skeletal muscle cells with high myogenic capacities. Mol Ther J Am Soc Gene Ther 2009;17:1948-1958.

10 Pisani DF, Clement N, Loubat A, Plaisant M, Sacconi S, Kurzenne J-Y, Desnuelle C, Dani C, Dechesne CA: Hierarchization of myogenic and adipogenic progenitors within human skeletal muscle. Stem Cells 2010;28:2182-2194.

11 Pisani DF, Dechesne CA, Sacconi S, Delplace S, Belmonte N, Cochet O, Clement N, Wdziekonski B, Villageois AP, Butori C, Bagnis C, Di Santo JP, Kurzenne JY, Desnuelle C, Dani C: Isolation of a highly myogenic CD34negative subset of human skeletal muscle cells free of adipogenic potential. Stem Cells 2010;28:753-764.

12 Lecourt S, Marolleau J-P, Fromigué O, Vauchez K, Andriamanalijaona R, Ternaux B, Lacassagne MN, Robert I, Boumédiene K, Chéreau F, Marie P, Larghéro J, Fiszman M, Vilquin JT: Characterization of distinct mesenchymal-like cell populations from human skeletal muscle in situ and in vitro. Exp Cell Res 2010;316:2513-2526.

13 Arrighi N, Moratal C, Clément N, Giorgetti-Peraldi S, Peraldi P, Loubat A, Kurzenne JY, Dani C, Chopard A, Dechesne CA: Characterization of adipocytes derived from fibro/adipogenic progenitors resident in human skeletal muscle. Cell Death Dis 2015; DOI:10.1038/cddis.2015.79.

14 Moratal C, Raffort J, Arrighi N, Rekima S, Schaub S, Dechesne CA, Chinetti G, Dani C: IL-1ß- and IL4-polarized macrophages have opposite effects on adipogenesis of intramuscular fibro-adipogenic progenitors in humans. Sci Rep 2018;8:17005.

15 Uezumi A, Fukada S, Yamamoto N, Ikemoto-Uezumi M, Nakatani M, Morita M, Yamaguchi A, Yamada H, Nishino I, Hamada Y, Tsuchida K: Identification and characterization of PDGFR $\alpha+$ mesenchymal progenitors in human skeletal muscle. Cell Death Dis 2014; DOI:10.1038/cddis.2014.161.

16 Natarajan A, Lemos DR, Rossi FMV: Fibro/adipogenic progenitors: a double-edged sword in skeletal muscle regeneration. Cell Cycle 2010;9:2045-2046.

17 Judson RN, Zhang RH, Rossi FMA: Tissue-resident mesenchymal stem/progenitor cells in skeletal muscle: collaborators or saboteurs? FEBS J 2013;280:4100-4108.

18 Chapman MA, Meza R, Lieber RL: Skeletal muscle fibroblasts in health and disease. Differentiation 2016;92:108-115.

19 Wang Y, Wehling-Henricks M, Samengo G, Tidball JG: Increases of M2a macrophages and fibrosis in aging muscle are influenced by bone marrow aging and negatively regulated by muscle-derived nitric oxide. Aging Cell 2015;14:678-688.

20 Hogrel J-Y, Barnouin Y, Azzabou N, Butler-Browne G, Voit T, Moraux A, Leroux G, Behin A, McPhee JS, Carlier PG: NMR imaging estimates of muscle volume and intramuscular fat infiltration in the thigh: variations with muscle, gender, and age. Age Dordr Neth 2015; DOI:10.1007/s11357-015-9798-5.

21 Skuk D, Goulet M, Roy B, Chapdelaine P, Bouchard JP, Roy R, Dugré FJ, Sylvain M, Lachance JG, Deschênes L, Senay H, Tremblay JP: Dystrophin expression in muscles of duchenne muscular dystrophy patients after high-density injections of normal myogenic cells. J Neuropathol Exp Neurol 2006;65:371-386. 


\section{Cellular Physiology Cell Physiol Biochem 2019;53:1029-1045 \begin{tabular}{l|l|l|l|l}
\hline DOI: 10.33594/000000196 2019 The Author(s). Published by &
\end{tabular} and Biochemistry Published online: 23 December 2019 Cell Physiol Biochem Press GmbH\&Co. KG \\ Moratal et al.: Myoblast Effects on Fibro-Adipogenic Progenitors}

22 Mozzetta C, Consalvi S, Saccone V, Tierney M, Diamantini A, Mitchell KJ, Marazzi G, Borsellino G, Battistini L, Sassoon D, Sacco A, Puri PL: Fibroadipogenic progenitors mediate the ability of HDAC inhibitors to promote regeneration in dystrophic muscles of young, but not old Mdx mice. EMBO Mol Med 2013;5:626639.

23 Lemos DR, Babaeijandaghi F, Low M, Chang CK, Lee ST, Fiore D, Zhang RH, Natarajan A, Nedospasov SA, Rossi FM: Nilotinib reduces muscle fibrosis in chronic muscle injury by promoting TNF-mediated apoptosis of fibro/adipogenic progenitors. Nat Med 2015;21:786-794.

24 Muñoz-Cánoves P, Serrano AL: Macrophages decide between regeneration and fibrosis in muscle. Trends Endocrinol Metab TEM 2015;26:449-450.

25 Kang X, Yang MY, Shi YX, Xie MM, Zhu M, Zheng XL, Zhang CK, Ge ZL, Bian XT, Lv JT, Wang YJ, Zhou BH, Tang KL: Interleukin-15 facilitates muscle regeneration through modulation of fibro/adipogenic progenitors. Cell Commun Signal 2018;16:42.

26 Kopinke D, Roberson EC, Reiter JF: Ciliary Hedgehog Signaling Restricts Injury-Induced Adipogenesis. Cell 2017;170:340-351.

27 Kuswanto W, Burzyn D, Panduro M, Wang KK, Jang YC, Wagers AJ, Benoist C, Mathis D: Poor Repair of Skeletal Muscle in Aging Mice Reflects a Defect in Local, Interleukin-33-Dependent Accumulation of Regulatory T Cells. Immunity 2016;44:355-367.

28 Vilquin JT, Marolleau JP, Sacconi S, Garcin I, Lacassagne MN, Robert I, Ternaux B, Bouazza B, Larghero J, Desnuelle C: Normal growth and regenerating ability of myoblasts from unaffected muscles of facioscapulohumeral muscular dystrophy patients. Gene Ther 2005;12:1651-1662.

29 Fontaine C, Cousin W, Plaisant M, Dani C, Peraldi P: Hedgehog signaling alters adipocyte maturation of human mesenchymal stem cells. Stem Cells 2008;26:1037-1046.

30 Straface G, Aprahamian T, Flex A, Gaetani E, Biscetti F, Smith RC, Pecorini G, Pola E, Angelini F, Stigliano E, Castellot JJ Jr, Losordo DW, Pola R: Sonic hedgehog regulates angiogenesis and myogenesis during postnatal skeletal muscle regeneration. J Cell Mol Med 2009;13:2424-2435.

31 Fiore D, Judson RN, Low M, Lee S, Zhang E, Hopkins C, Xu P, Lenzi A, Rossi FM, Lemos DR: Pharmacological blockage of fibro/adipogenic progenitor expansion and suppression of regenerative fibrogenesis is associated with impaired skeletal muscle regeneration. Stem Cell Res 2016;17:161-169.

32 Mueller AA, van Velthoven CT, Fukumoto KD, Cheung TH, Rando TA: Intronic polyadenylation of PDGFR $\alpha$ in resident stem cells attenuates muscle fibrosis. Nature 2016;540:276-279.

33 Margoni A, Fotis L, Papavassiliou AG: The transforming growth factor-beta/bone morphogenetic protein signalling pathway in adipogenesis. Int J Biochem Cell Biol 2012;44:475-479.

34 Zaragosi LE, Wdziekonski B, Villageois P, Keophiphath M, Maumus M, Tchkonia T, Bourlier V, MohsenKanson T, Ladoux A, Elabd C, Scheideler M, Trajanoski Z, Takashima Y, Amri EZ, Lacasa D, Sengenes C, Ailhaud G, Clément K, Bouloumie A, Kirkland JL, et al.: Activin a plays a critical role in proliferation and differentiation of human adipose progenitors. Diabetes 2010;59:2513-2521.

35 Dani C: Activins in adipogenesis and obesity. Int J Obes 2013;37:163-166.

36 Meng XM, Tang PMK, Li J, Lan HY: TGF- $\beta$ /Smad signaling in renal fibrosis. Front Physiol 2015; DOI:0.3389/ fphys.2015.00082.

37 Ohga E, Matsuse T, Teramoto S, Katayama H, Nagase T, Fukuchi Y, Ouchi Y: Effects of activin A on proliferation and differentiation of human lung fibroblasts. Biochem Biophys Res Commun 1996;228:391396.

38 Weiss A, Attisano L: The TGFbeta superfamily signaling pathway. Wiley Interdiscip Rev Dev Biol 2013;2:4763.

39 Robbins DJ, Fei DL, Riobo NA: The Hedgehog signal transduction network. Sci Signal 2012; DOI:10.1126/ scisignal.2002906.

40 Hui CC, Angers S: Gli proteins in development and disease. Annu Rev Cell Dev Biol 2011; DOI:10.1146/ annurev-cellbio-092910-154048.

41 Dennler S, André J, Alexaki I, Li A, Magnaldo T, ten Dijke P, Wang XJ, Verrecchia F, Mauviel A: Induction of sonic hedgehog mediators by transforming growth factor-beta: Smad3-dependent activation of Gli2 and Gli1 expression in vitro and in vivo. Cancer Res 2007;67:6981-6986. 


\section{Cellular Physiology Cell Physiol Biochem 2019;53:1029-1045

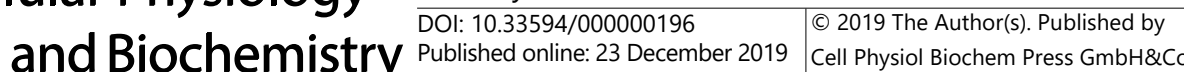 \\ Moratal et al.: Myoblast Effects on Fibro-Adipogenic Progenitors}

42 Nye MD, Almada LL, Fernandez-Barrena MG, Marks DL, Elsawa SF, Vrabel A, Tolosa EJ, Ellenrieder V, Fernandez-Zapico ME: The transcription factor GLI1 interacts with SMAD proteins to modulate transforming growth factor $\beta$-induced gene expression in a p300/CREB-binding protein-associated factor (PCAF)-dependent manner. J Biol Chem 2014;289:15495-15506.

43 Javelaud D, Pierrat M-J, Mauviel A: Crosstalk between TGF- $\beta$ and hedgehog signaling in cancer. FEBS Lett 2012;586:2016-2025.

44 Lukjanenko L, Karaz S, Stuelsatz P, Gurriaran-Rodriguez U, Michaud J, Dammone G, Sizzano F, Mashinchian O, Ancel S, Migliavacca E, Liot S, Jacot G, Metairon S, Raymond F, Descombes P, Palini A, Chazaud B, Rudnicki MA, Bentzinger CF, Feige JN: Aging Disrupts Muscle Stem Cell Function by Impairing Matricellular WISP1 Secretion from Fibro-Adipogenic Progenitors. Cell Stem Cell 2019;24:433-446.

45 Ikemoto-Uezumi M, Uezumi A, Tsuchida K, Fukada S, Yamamoto H, Yamamoto N, Shiomi K, Hashimoto N: Pro-Insulin-Like Growth Factor-II Ameliorates Age-Related Inefficient Regenerative Response by Orchestrating Self-Reinforcement Mechanism of Muscle Regeneration. Stem Cells 2015;33:2456-2468.

46 Ogawa R, Ma Y, Yamaguchi M, Ito T, Watanabe Y, Ohtani T, Murakami S, Uchida S, De Gaspari P, Uezumi A, Nakamura M, Miyagoe-Suzuki Y, Tsujikawa K, Hashimoto N, Braun T, Tanaka T, Takeda S, Yamamoto $\mathrm{H}$, Fukada S: Doublecortin marks a new population of transiently amplifying muscle progenitor cells and is required for myofiber maturation during skeletal muscle regeneration. Dev Camb Engl 2015; DOI:10.1242/ dev.122317.

47 Brack AS, Conboy MJ, Roy S, Lee M, Kuo CJ, Keller C, Rando TA: Increased Wnt signaling during aging alters muscle stem cell fate and increases fibrosis. Science 2007;317:807-810.

48 Brioche T, Pagano AF, Py G, Chopard A: Muscle wasting and aging: Experimental models, fatty infiltrations, and prevention. Mol Aspects Med 2016;50:56-87.

49 Juban G, Saclier M, Yacoub-Youssef H, Kernou A, Arnold L, Boisson C, Ben Larbi S, Magnan M, Cuvellier S, Théret M, Petrof BJ, Desguerre I, Gondin J, Mounier R, Chazaud B: AMPK Activation Regulates LTBP4Dependent TGF- $\beta 1$ Secretion by Pro-inflammatory Macrophages and Controls Fibrosis in Duchenne Muscular Dystrophy. Cell Rep 2018; DOI:10.1016/j.celrep.2018.10.077. 